\title{
DATOS SOBRE LA ALIMENTACION DE LA LECHUZA (Tyto alba) EN VALENCIA
}

\author{
por \\ $\begin{array}{lllll}\text { J. MARTIN } & \text { Y J. R. VERICAD } & \\ & & \end{array}$
}

\section{INTRODUCCION}

Si bien la dieta de la lechuza es bastante conocida desde varios puntos de vista en distintos países europeos y en algunas zonas de Iberia, donde en su parte nor-oriental cabe destacar la labor de Sans-Coma, la gran laguna que en ese aspecto supone en la bibliografia consultada el Levante ibérico, nos ha animado a dar a conocer los resultados del estudio de varios lotes de egagrópilas de lechuza provenientes básicamente de la provincia valenciana.

\section{MATERIAL Y METODOS}

El total de egagrópilas recolectadas y analizadas es de un millar, alcanzando 3.977 individuos el total de presas vertebradas, que provienen de nueve localidades valencianas y tres de Alicante, cuyas características y situación vienen dadas en la tabla 1.

Los resultados están expresados en porcentaje numérico de presencia para cada especie y localidad y en porcentaje de biomasa de peso seco para cada especie y localidad. El cálculo de biomasa de peso seco aportado por cada especie presa se ha hecho basándose en los pesos secos obtenidos para cada especie presa por Vericad et al. (1976).

Este trabajo contenía originalmente una parte dedicada al número de presas por egagrópila y su relación con la especie o especies depredadas y categoria de edad de las mismas en un lote de 200 ovillos, cuya publicación se ha omitido por coincidir plenamente con lo expuesto por Sans-Coma y Kahmann (1976).

1. Dpto. de Biología y Geología. C.E.U. Alicante.

2. Centro pirenaico de Biología experimental. Jaca (Huesca). 


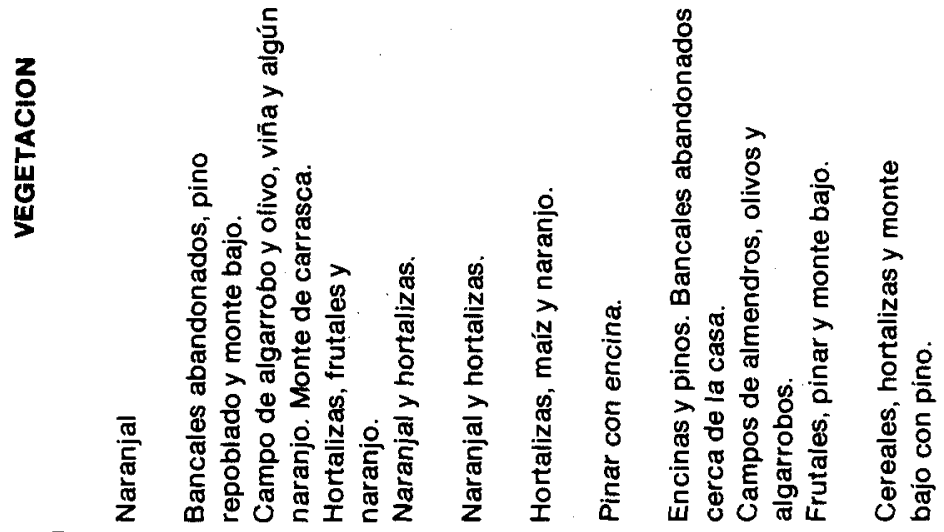

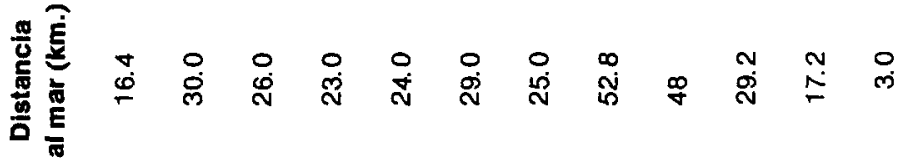

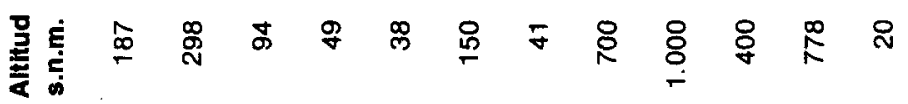

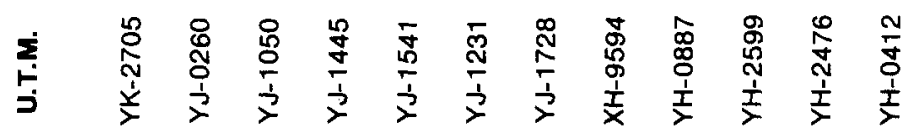

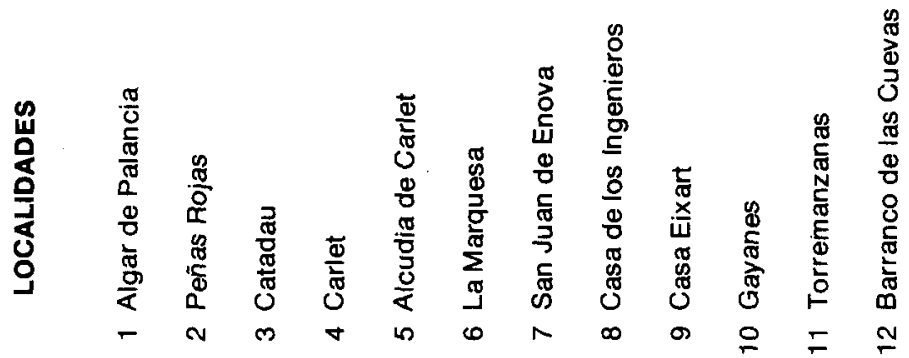




\section{RESULTADOS}

1) Alimentación de la lechuza en las localidades prospectadas.

En la tabla II se presenta el espectro trófico de $T$. alba en cada una de las localidades en porcentaje numérico y de biomasa de peso seco de cada tipo de presa considerado para los cálculos, a nivel de clase en anfibios, reptiles y aves, a nivel de orden en quirópteros y a nivel de especie en el resto de mamíferos.

ANFIBIOS: Representan el $4.5 \%$ del total de las presas vertebradas del conjunto de todas las localidades. Se citan en cinco de las doce localidades $\left(n .{ }^{\circ} 3,4,5,6\right.$ y 12). Su aportación numérica a la dieta de la lechuza varía entre $0.6 \%$ y $13,3 \%$, mientras que desde el punto de vista energético su valor disminuye ( $0.4 \%$ a $8.6 \%$ ).

Las especies implicadas son Rana rídibunda, Pelodytes punctatus y Pelobates cultripes, siendo la primera la que se presenta en mayor porcentaje. Las localidades que acusan la presencia de Anfibios corresponden a lugares de baja altitud atravesados por ríos y acequias. Debido a sus hábitos principalmente nocturnos, los Anfibios, en las localidades donde abundan, son depredados más o menos activamente. En Europa (Schmidt, 1973), como especie-presa se encuentran diferentes especies de Rana así como Pelobates fuscus. En Andalucia occidental Valverde (1967) y Herrera (1973) encuentran que Pelobates cultripes, especie considerada por Guérin (1928) como no depredada por venenosa, es una presa común así como Rana ridibunda y Pelodytes punctatus. Anuros con abundantes secreciones tóxicas como las dos especies de Bufo e Hyla parecen ser ignoradas por la lechuza, aunque pueden formar parte muy esporádicamente en su dieta (Schmidt, 1973).

REPTILES: Representan el $3.11 \%$ del total de presas vertebradas y se presentan en siete de las doce localidades $\left(n .0^{\circ} 1,3,4,5,6,7\right.$ y 12). El porcentaje numérico varía entre el $0.2 \%$ y el $15.3 \%$ y el porcentaje de biomasa aportada en peso disminuye en importancia oscilando entre $0.1 \%$ y $5 \%$.

Las presas más representativas son los Geckónidos (Tarentola mauritanica y Hemydactylus turcicus) y en la muestra $n .^{\circ} 7$ aparecen además Lacerta hispanica y Psammodromus hispanicus. Parece inevitable relacionar la abundancia de Geckónidos en egagrópilas con la actividad nocturna de estos saurios asi como con la proximidad a núcleos urbanos de tierras bajas. Aquí se presenta el problema ya expuesto por Herrera (1973) de coincidencia temporal del depredador (la lechuza) que es básicamente nocturno, con sus presas potenciales (los saurios) de actividad diurna. Los Geckónidos con parte de actividad crepuscular y nocturna (Martínez Rica, 1974) son lógica- 


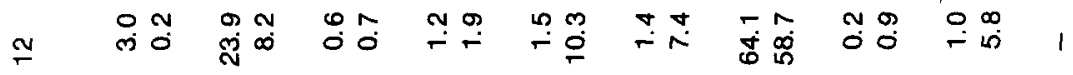

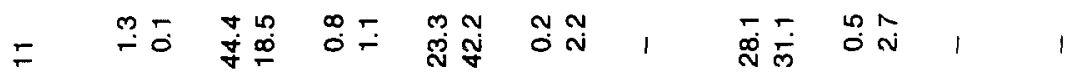

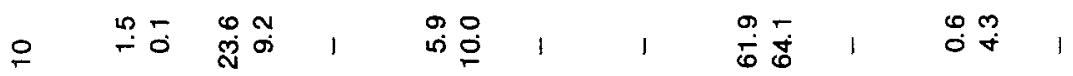

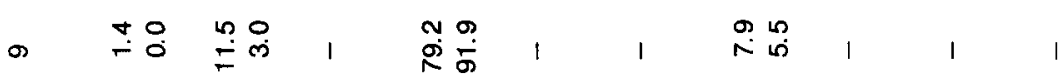

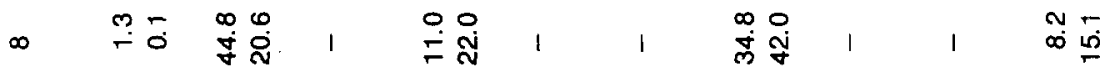

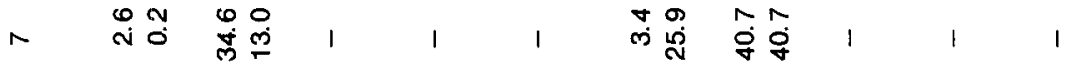

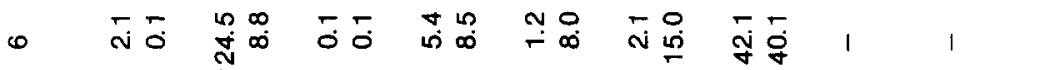

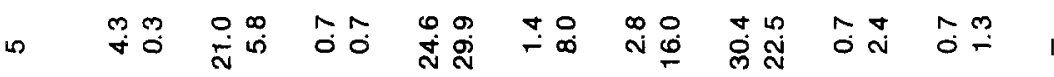

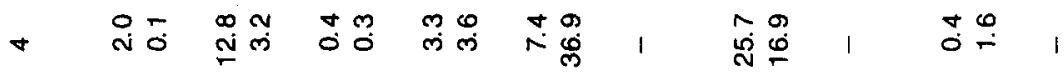

$m$ ํㅣ ลิำ

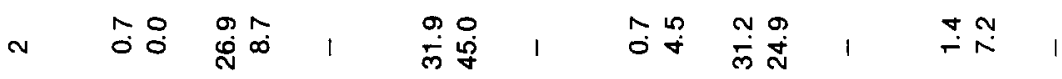

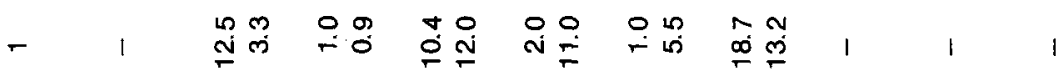

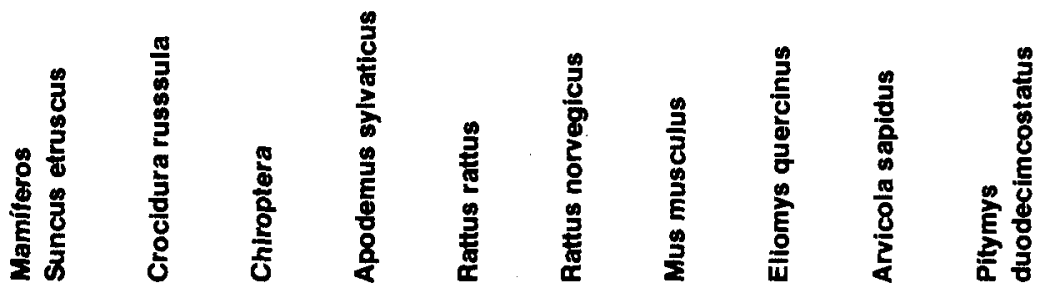




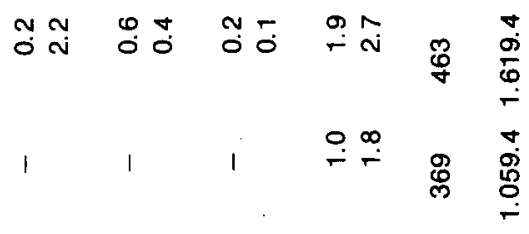

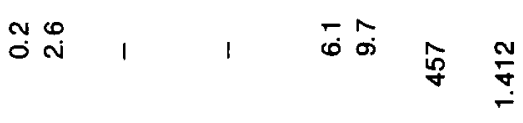

赵

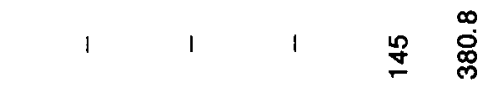

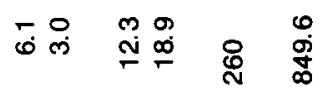

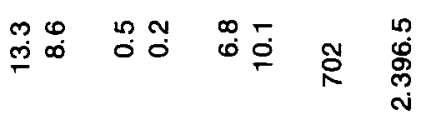

mヘ

ๆ

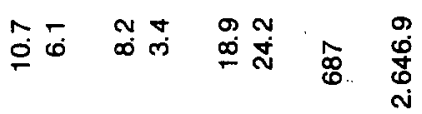

유

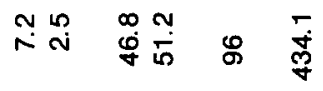

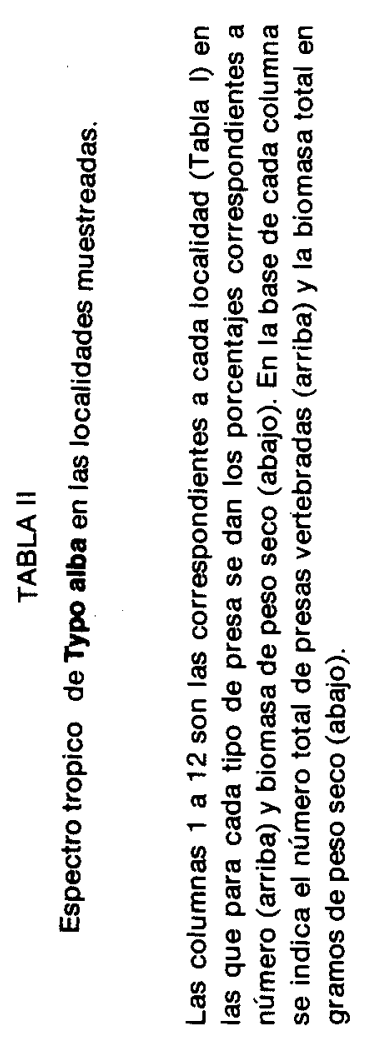

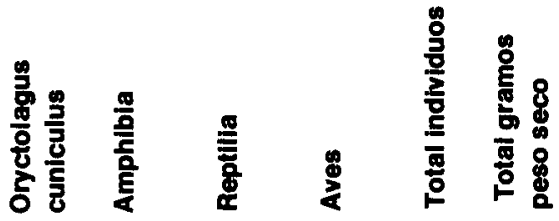


mente los depredados con mayor intensidad. Nos (1960) para la localidad catalana de Orrius, Herrera (1973) para Andalucia occidental y Martínez Rica (1974) para Mallorca señalan sucesivamente la depredación que sobre cualquiera de las dos especies de Geckónidos efectúa la lechuza. Para el resto de los saurios hay datos de depredación sobre Lacerta viridis (Guérin, 1928), Lacerta sicula (Witte, 1964), Psammodromus algirus y Acanthodactylus erythurus (Valverde, 1967) y Psammodromus sp., Lacerta sp. y Lacerta lepida (Herrera, 1973), si bien de todos estos estudios se deduce que estos saurios diurnos representan un porcentaje bajísimo tanto numérico como en biomasa. Sin embargo, según Rey y Rey (1974), en Ibiza, los reptiles en conjunto como presas, respecto a todos los vertebrados depredados por la lechuza alcanzan el 13.1\%, donde deben tener sin duda bastante importancia.

AVES: Representa el $9.85 \%$ del total de las presas vertebradas. Se presentan en diez localidades (sólo ausentes en la n. 8 y 9). El porcentaje numérico muestra mayor variabilidad que en los grupos anteriores. Está comprendido entre el $1 \%$ en la localidad n. ${ }^{\circ} 11$ y el 46.8\% en la n. ${ }^{\circ} 1$.

La importancia de este grupo se ve incrementada en el porcentaje de biomasa aportada variando entre $1.8 \%$ en la localidad $n .{ }^{\circ} 11$ y el $51.2 \%$ en la $n .^{\circ} 1$. Las localidades que contribuyen en mayor grado al porcentaje total de biomasa de vertebrados, en cuanto a aves, se refiere, son las del naranjal $\left(n .{ }^{\circ} 1,3,4,5,6\right.$ y 7$)$ con una media del 24\%. Significativa es la ausencia total de aves en las localidades de altitud (.$\circ 8$ y 9 ).

La identificación de cada una de las especies, por carecer de una colección apropiada de cráneos, no ha sido posible. La presa más abundante es Passer domesticus y destaca su presencia en las zonas antrópicas (localidades n. ${ }^{\circ} 1,3$ y 4). Otras especies implicadas son Carduelis sp., Turdus sp. y Galerida sp.

La fracción ornítica de la dieta de las estrígidas ha sido casi siempre dejada de lado. En nuestro país, Valverde (1967) y Herrera (1973) detallan la lista de especies de aves o familias de aves depredadas por la lechuza en Andalucía. Sans-Coma (1974) hace lo mismo para las localidades catalanas estudiadas por él. Autores extranjeros como Schmidt $(1968,1972)$ estudian con detalle la alimentación ornítica de la lechuza en Hungría. La principal conclusión que se puede extraer, es que el gorrión común (Passer domesticus) constituye una parte muy importante del total de aves depredadas como se ve claramente en el cuadro resumen ofrecido por Schmidt (1973), a menos que la lechuza tenga por territorios de caza biotopos algo especiales o marginales (marismas) o lugares alejados de zonas antrópicas. Schmidt, (1972) encuentra que hay una 
correlación negativa bastante estricta entre el porcentaje de gorrión común respecto al total de aves depredadas y la altitud.

MAMIFEROS: Este grupo representa el porcentaje más importante, tanto numéricamente (82.49\%) como en biomasa aportada (82.32\%) con relación al número total de presas vertebradas del conjunto de las localidades.

El número total y porcentaje numérico de presas de los órdenes Insectivora y Rodentia, vienen expresados en la tabla resumen siguiente:

\section{LOCALIDADES}

$\begin{array}{llllllllllll}1 & 2 & 3 & 4 & 5 & 6 & 7 & 8 & 9 & 10 & 11 & 12\end{array}$

INSECTIVORA

$\begin{array}{lllllllllllll}\text { n. } & 12 & 39 & 170 & 36 & 35 & 190 & 97 & 67 & 36 & 115 & 169 & 125\end{array}$

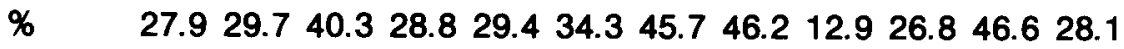

RODENTIA

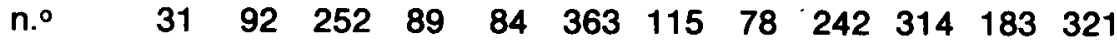

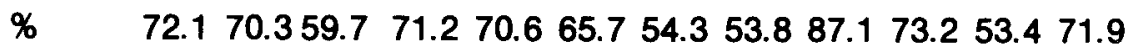

$\begin{array}{lllllllllllllllll}\text { I/R } & 0.38 & 0.42 & 0.67 & 0.40 & 0.41 & 0.52 & 0.84 & 0.85 & 0.14 & 0.36 & 0.87 & 0.38\end{array}$

El cociente entre los porcentajes numéricos de estos dos grupos (I/R) oscila entre 0.14 en la localidad $n .{ }^{\circ} 9$ y 0.97 en la $n .{ }^{\circ} 11$.

La dominancia de Roedores sobre Insectívoros se hace patente exceptuando las localidades $n .^{\circ} 7,8$ y 11 , en que la diferencia es menor de un $10 \%$. En cuando a la biomasa que aporta cada grupo, la contribución de Insectívoros es mínima frente a la de Roedores. En la mayoría de las localidades, el porcentaje numérico de Insectívoros se halla comprendido entre el $25 \%$ y el $35 \%$, datos que coinciden con los expuestos y resumidos por Herrera (1973) para varios países europeos. 
Recientes datos de Rey y Rey (1974) sobre la dieta de la lechuza en Ibiza hacen suponer, en cambio, una mayor importancia de los insectivoros mientras que en otras islas mediterráneas, Creta (Cheyllan, 1976) y Córcega (Kahmann y Brotzler, 1956; Charvin, 1974) su impacto es más variable.

Las dos únicas especies de insectívoros implicadas como presa son Crocidura russula y Suncus etruscus, presentes las dos en todas las localidades excepto Suncus etruscus en la n. ${ }^{\circ} 1$. El porcentaje numérico respecto al total de presas vertebradas de Crocidura russula varía entre $11.5 \%$ y $44.8 \%$, siendo la media de $25.26 \%$. El porcentaje de biomasa varía entre el $3 \%$ y el $20.6 \%$ con una media de 9.11\%. Suncus etruscus presenta un porcentaje numérico sobre el total de presas vertebradas que varía entre el $0.7 \%$ y el $4.3 \%$ siendo la media de $2.01 \%$. En cuando al porcentaje de biomasa que aporta es muy escaso, no sobrepasando nunca el $0.3 \%$. En todas las localidades, el número de Crocidura es superior al de Suncus.

Los quirópteros aparecen en siete localidades y el porcentaje, tanto numérico como de biomasa aportada no excede del $1.1 \%$, lo que no supone apenas aportación al régimen alimenticio de Tyto alba. Como especies depredadas se encuentran Rhinolophus ferrumequinum y Pipistrellus sp.

Los quirópteros, por su potencia de vuelo, son más difícilmente alcanzables por la lechuza que las presas epígeas, por lo que parece que su captura es más bien ocasional y depende de circunstancias favorables. Así, Bauer (1956) encontró que los quirópteros formaban el 55.3\% de la dieta de la lechuza en una localidad alemana, pero esto no es más que una especialización individual en condiciones determinadas.

Normalmente, el papel de los quirópteros en la dieta de la lechuza es poco importante como se desprende de nuestros datos y de los correspondientes a España sudoccidental de Valverde (1967) y Herrera (1973). Referente a Cataluña, Nadal y Palaus (1967) encuentran quirópteros en la dieta de la lechuza pero aún en porcentajes menores y Sans-Coma (1974) destaca la ausencia de quirópteros en sus lotes de egagrópilas de lechuza. Uno de los autores (Vericad) en más de 10.000 presas de lechuza contabilizadas del Alto Aragón occidental, encuentra sólo una mandíbula de quiróptero. En la isla de Córcega (Kahmann y Brotzler, 1956) los murciélagos no representan más que escasamente el $1 \%$ y Rey y Rey (1974) dan para la isla de Ibiza sólo el $0.1 \%$ de quirópteros respecto al total de presas vertebradas.

Los roedores representan el $54.63 \%$ del total de presas vertebradas y el $72.62 \%$ de la biomasa total. 
Son dos las especies (Apodemus sylvaticus y Mus musculus) cuya presencia es constante, ya que no están estrechamente localizadas a ningún biotopo especial teniendo una distribución difusa y una fuerte densidad (Heim de Balsac y de Beaufort, 1966). De ellas la más abundante es Mus musculus con un porcentaje numérico sobre el total de presas vertebradas que varía desde el $7.9 \%$ al $64.1 \%$ y con un porcentaje de biomasa del $5.5 \%$ al $64.1 \%$. Le sigue en importancia en orden a su porcentaje numérico, Apodemus sylvaticus que varía entre $1.2 \%$ y $79.2 \%$ respecto al total de presas vertebradas y con un porcentaje de biomasa que oscila de $1.9 \%$ a $91.9 \%$. Estas dos especies frente al número total de Roedores representan un $94 \%$ y un $73.6 \%$ de la biomasa aportada por éstos. El alto porcentaje de Mus es debido sin duda a la clara influencia mediterránea por un lado y antropógena por otro, de la región estudiada.

Otras dos especies que se pueden considerar habituales en el régimen alimenticio de la lechuza son Rattus rattus y Rattus inorvegicus. La presencia de una o de las dos especies es constante en todas las localidades exceptuando las $n .{ }^{\circ} 8,9$ y 10 . El porcentaje numérico sobre el total de presas vertebradas varía entre $0.2 \%$ a $7.4 \%$ para Rattus rattus, y de $0.7 \%$ a $3.4 \%$ para Rattus norvergicus. La importancia de estas especies a la dieta de la lechuza se ve aumentada si nos referimos a la biomasa total aportada, variando los porcentajes entre $2.2 \%$ y $36.9 \%$ para Rattus rattus y entre $4.5 \%$ a $25.99 \%$ en Rattus norvegicus. Respecto al número total de roedores, representan un $4.6 \%$ y un $23.3 \%$ en relación a la biomasa aportada por dichos roedores. Dichas especies están más localizadas en biotopos particulares mostrando un carácter más antopófilo que las anteriores (Heim de Balsac, 1966). La presencia de Rattus norvegicus; parece estar muy unida a localidades con ríos o acequias en nuestro caso y en general (S. - Girons, 1974).

Arvicola sapidus y Eliomys quercinus están representados minimamente debido a que su localización contagiosa está unida a biotopos particulares como cursos de agua o estanques en Arvicola o bosques y roquedos en Eliomys (S. - Girons et al. 1974). En los porcentajes numéricos sobre el total de presas vertebradas no superan el $1.4 \%$ acentuándose un poco su porcentaje en cuanto a biomasa aportada. Sobre el número total de roedores, Arvicola sapidus representa el $0.55 \%$ y Eliomys quercinus el $0.27 \%$ y en cuanto a la biomasa aportada por roedores, el primero de ellos figura con un $1.64 \%$ y el segundo con un $0.84 \%$.

Pitymys duodecimcostatus, presa frecuente en todos los lotes de egagrópilas estudiadas en nuestro pais, aparece aquí únicamente en la localidad más interior y de mayor altitud ( $\left.n .{ }^{\circ} 8\right)$. Su porcentaje numérico es de $8.2 \%$ respecto al total de presas vertebradas de esa 
localidad. El hecho ya resaltado anteriormente (Vericad et al. 1976) de la poca importancia de $P$. duodecimcostatus en la dieta de $T$. alba en el Levante ibérico puede ser bien reflejo de una baja densidad o bien a la pluviosidad, la cual determina su presencia como presa demostrado ya por Sans-Coma y Kahmann (1976) y fue sugerido algo antes por Saint-Girons y Martin (1973) para P. subterraneus.

Oryctolagus cuniculus, presa extremadamente ocasional en la dieta de la lechuza, en nuestro estudio se cita en dos localidades; los dos cráneos encontrados pertenecen a individuos juveniles. Valverde (1967) lo cita dentro del régimen de la lechuza y Herrera (1973), de un total de 14.168 presas vertebradas encuentra dos individuos juveniles de esta especie.

2) Correlaciones de algunos parámetros de las localidades con la dieta de la lechuza.

En este apartado se pretende caracterizar con un nivel de significación adecuado el grado de correlación simple existente entre una de dos determinadas variables de las localidades (altitud y distancia mínima al mar) y las diversidades de presas en relación a la biomasa en peso seco aportada y número de individuos respecto al taxón clase (calculadas aquellas diversidades mediante la fórmula propuesta por LLoyd y Ghelardi, 1964), asi como correlación de aquellas variables con los porcentajes numéricos y de biomasa, referidos tanto a especie como a taxones de orden superior. En total se han calculado 25 correlaciones de las cuales sólo 12 han superado un nivel mínimo de significación y se presentan en la tabla III, utilizándose para ello los datos del presente trabajo y los referidos a la provincia de Alicante expuestos por Vericad et al. (1976), elevándose así a 24 el número de localidades de recogida de egagrópilas con un total de 10.547 presas vertebradas.

\section{RESUMEN Y CONCLUSIONES}

El estudio de nueve lotes de egagrópilas de lechuza provenientes de Valencia y tres de Alicante que han dado un total de 3.977 presas vertebradas confirman las conclusiones expresadas en trabajo anterior (Vericad et al. 1976) referidos a ovillos de aquella especie en quince localidades alicantinas. Ha quedado patente el impacto que tienen las aves (Passer domesticus, básicamente) y determinadas especies mamíferas como Crocidura russula, Mus musculus y Apodemus sylvaticus en la dieta de la lechuza. Otras especies como Suncus etruscus y las dos del género Rattus se presentan siempre en bajo porcentaje respecto al total de presas vertebradas pero de manera constante en casi todas las localidades estudiadas. Arvicola sapidus y 
Distancia minima al mar / \% numérico de aves respecto a total de presas.

$\begin{array}{ll}-0.3296 & 0.1\end{array}$

Distancia mínima al mar / \% numérico de Apodemus en número total de presas.

$0.4551 \quad 0.02$

Altitud / diversidad biomasa por grandes grupos taxonómicos.

Altitud / diversidad numérica por grandes grupos taxonómicos.

Altitud / $\%$ biomasa mamífera en biomasa total.
0.3787
0.1

Altitud / \% numérico de mamíferos respecto a total de presas.

$0.5007 \quad 0.01$

Altitud / \% numérico de anfibios y reptiles respecto a total de presas.

$-0.4017 \quad 0.05$

Altitud / \% numérico Apodemus respecto a total de presas.

$0.6334 \quad 0.001$

Altitud / \% numérico Apodemus en. número total de mamíferos.

$0.5965 \quad 0.005$

Altitud / \% numérico de Mus en número total de mamíferos.

Altitud / \% numérico Rattus en número total de mamíferos.

$-0.5886 \quad 0.005$

Altitud / \% biomasa Rattus en biomasa total.

$-0.6303 \quad 0.001$

\section{Tabla III}

Valores del coeficiente de correlación $r$ y nivel de significación del mismo entre las variables indicadas. 
Eliomys quercinus así como los quirópteros apenas cuentan en cuanto al número y biomasa en el menú de Tyto alba, lo mismo que determinadas especies de anfibios y reptiles las cuales, sin embargo, constituyen un adecuado complemento. Los topillos (Pitymys) tampoco constituyen aquí presa importante.

La correlación significativa existente entre la altitud y el régimen de la lechuza con los datos aportados en este trabajo sobre la provincia de Valencia y en trabajo anterior citado más arriba sobre la de Alicante, resulta que el porcentaje de anfibios, reptiles y aves respecto al total de presas vertebradas, se correlaciona negativamente con la altitud, así como la diversidad trófica en relación a la biomasa aportada por grandes grupos taxonómicos y la diversidad trófica en relación al número de individuos por cada grupo taxonómico. Por el contrario hay correlación positiva con la altitud del porcentaje de mamíferos respecto al total de presas vertebradas. Las especies del género Rattus y Mus musculus, al contrario que Apodemus, disminuyen en porcentaje con la altitud, siendo la lechuza en la región, considerada más estenófaga con la altitud.

\section{BIBLIOGRAFIA}

BAUER, K. - 1956. Schleiereule (Tyto alba Scop.) als Fledermausjäger. J.f. Orn. 97 (3): 335-340.

CHARVIN, G. - 1974. Analyse de 60 pelottes de réjection de Chouette effraie Tyto alba provenant de Corse. Jean Le Blanc 13: 21.

CHEYLAN, G. - 1976. Le régime alimentaire de la Chouette effraie Tyto alba en Europe méditerranéénne. La Terre ot la Vle, 30 (4): 565-579.

GUERIN, G. - 1928. Rógime et crolssance de l'Effraye commune en Vendée. Lechevalier. París.

HEIM DE BALSAC, H. y F. DE BEAUFORT. - 1976. Régime alimentaire de l'Effraie dans les Bas-Dauphine. Applications à l'étude des Vertébres. Alanda 34: 309-324.

HERRERA, C. M. - 1973. Régimen alimenticio de Tyto alba en España suroccidental. Ardeola, 19 (2): 359-394.

KAHMANN, H. y A. BROTZLER. - 1956. Die Ernährung der Schleiereule (Tyto alba) und dans Bild der Verbreitung kleiner Säugetiere auf der Insel Korsika. Blol. Zbl. Lelpzlg. 76: 67-83.

LLOYD, M. y R. J. GHELARDI. - 1964. A table for calculating the requitability component of species diversity. J. Anịm. Ecol. 33: 217-225.

MARTINEZ-RICA, J. P. - 1974. Contribución al estudio de la biología de los gecónidos ibéricos (Rep. Sauria) P. Cent. plr. Blol. exp., 5: 8-291. 
NADAL, J. y X. PALAUS. - 1967. Micromamíferos hallados en egagrópilas de Tyto alba. P. Inst. Blol. Apl. 42: 5-15.

NOS, R. - 1960. Estudio de los ovillos regurgitados por una pareja de Tyto alba en la comarca de Maresma (prov. Barcelona). Mlsc. Zool., 1: 3-10.

REY, J. C. y J. M. REY. - 1974. Nota preliminar sobre las musarañas del género Crocidura Wagler, 1832 en las islas Baleares. Bol. Est. C. Ecología, 3 (6): 79-85.

SAINT-GIRONS, M. - Ch. H. BAUDOIN, D. JAMMOT y J. CHALINE. - 1974. Les proles des Rapaces. Pettes mammitores of leur environment. Doin. Paris.

SAINT-GIRONS, M. - Ch. y Ch. MARTIN. - 1973. Adaptation du régime de quelques rapaces nocturnes au paysage rural. Les proies de l'Effraie et du Moyen-Duc dans le département de la Somme. Bull. Ecol. 4 (2): 95-120.

SANS-COMA, V. - 1970. Sobre la distribución de micromamíferos del N.E. de la península ibérica, con algunas consideraciones metodológicas. P. Inst. Blol. Apl. 48: 125-144.

SANS-COMA, V. - 1974. Sobre la alimentación de Tyto alba en la región continental catalana. Mlsc. Zool. 3 (4): 163-169.

SANS-COMA, V., T. CLARAMUNT y J. GOSALBEZ. - 1976. Die MittelmeerKleinwühlmaus, Pitymys duodecimcostatus (de Selys Longchamps, 1839) in der Ernährung der Schleiereule (Tyto alba) in Katalonien (Spanien). Säugetierk. Mitt. 24 (1): $77-79$.

SANS-COMA, V. y H. KAHMANN. - 1976. Quantitative Untersuchungen über die Ernährung der Schleiereule (Tyto alba) in Katalonien (Spanien). Săugetierk. Mitt. 24(1): 5-11.

SCHMIDT, E. - 1968. Der Haussperling (P. domestlcus) und der Feldsperling (P. montanus) als Nahrung der Schleiereule (Tyto alba) in Ungarn. Intern. Stud. on Sparrows, 2: 96-101.

SCHMIDT, E. - 1972. Uber die Vogelnahrung der Schleiereule Tyto alba und der Waldohreule (Aslo otus) in Ungarn. Ornis Fennica, 49: 98-102.

SCHMIDT, E. - 1973. Die Nahrung der Schleiereule (Tyto alba) in Europa. Z.f. Ang. Zool., 60 (1): 43-70.

VALVERDE, J. A. - 1967. Estructura de una comunidad de vertebrados terrestres. C.S.I.C.

VERICAD, J. R., A. ESCARRE y E. RODRIGUEZ. - 1976. Datos sobre la dieta de Tyto alba y Bubo bubo en Alicante (S.E. de Iberia). Mediterránea, 1: 49-59.

WITTE, G. - 1964. Zur Systematik der Insektenfresser des Monte-Gargano-Gabietes (Italien). Boon. Zool. Behr. 15: 1-35. 